\title{
Diseño y evaluación de una secuencia de enseñanza- aprendizaje STEAM para Educación Primaria
}

\author{
Ileana M. Greca (D), Jairo Ortiz-Revilla iD \\ Departamento de Didácticas Específicas, Facultad de Educación, Universidad de Burgos, Burgos, España. \\ imgreca@ubu.es,jortizr@ubu.es \\ Irene Arriassecq \\ ECienTec, Facultad de Ciencias Exactas, Universidad Nacional del Centro de la Provincia de Buenos Aires, \\ Argentina.irenearn@exa.unicen.edu.ar
}

[Recibido: 9 agosto 2020. Revisado: 28 octubre 2020. Aceptado: 1 diciembre 2020]

\begin{abstract}
Resumen: Este estudio muestra el diseño y la evaluación de una secuencia de enseñanza-aprendizaje (SEA) con enfoque STEAM integrado empleando la investigación basada en el diseño. La SEA se implementó en un total de seis grupos de alumnado de sexto de Educación Primaria $(\mathrm{N}=121)$, realizándose tres iteraciones con sucesivas ampliaciones de la muestra. Los resultados obtenidos de la evaluación competencial parecen indicar que el modelo teórico empleado en el diseño de la SEA es viable para el desarrollo competencial tanto científico como integral de los escolares.
\end{abstract}

Palabras clave: Competencia; Secuencias de enseñanza-aprendizaje; Investigación basada en el diseño; Educación STEAM integrada; Educación Primaria.

Design and evaluation of a STEAM teaching-learning sequence for primary education

Abstract: This study shows the design and evaluation of a teaching-learning sequence (TLS) with an integrated STEAM approach using design-based research. The TLS was implemented in a total of six groups of students in sixth grade of primary education $(\mathrm{N}=121)$, performing three iterations with successive extensions of the sample. The results obtained from the competence evaluation seem to indicate that the theoretical model used in the design of the SEA is viable for the scientific and integral competence development of students.

Keywords: Competence; Teaching-learning sequences; Design-based research; Integrated STEAM education; Primary education.

Para citar este artículo: Greca I. M., Ortiz-Revilla J. y Arriassecq I. (2021) Diseño y evaluación de una secuencia de enseñanza-aprendizaje STEAM para Educación Primaria. Revista Eureka sobre Enseñanza y Divulgación de las Ciencias 18(1), 1802. doi: 10.25267/Rev_Eureka_ensen_divulg_cienc.2021.v18.i1.1802

\section{Introducción}

La educación STEAM integrada (iSTEAM), con su adición de artes a las disciplinas STEM acrónimo en inglés de Ciencias, Tecnología, Ingeniería y Matemáticas-, es un enfoque complejo y discutido centrado en la resolución de problemas relevantes, cercanos al alumnado y con preferencia del uso de abordajes interdisciplinarios y transdisciplinarios. Por una parte, comparte una base común con los enfoques STEM que buscan, principalmente, incentivar vocaciones científico-tecnológicas. Por otra, contempla la inclusión de las artes, lo cual, según diversos autores, favorece la recuperación de objetivos y propósitos educativos orientados al desarrollo competencial integral, la inclusión social, la participación ciudadana o la sostenibilidad (entre otros, Colucci-Gray, Burnard, Gray y Cooke 2019, Ortiz-Revilla, Greca y Adúriz-Bravo 2018, Zeidler 2016). A pesar de que la implementación de iniciativas iSTEAM ha ido en aumento, los marcos teóricos existentes no desarrollan los principios teóricos con la especificidad suficiente para que los docentes los apliquen en la preparación de sus clases (Chu, Martin y Park 2019), y son muy escasos los modelos de instrucción concretos (Yakman 2008). Esto limita las oportunidades para que los docentes diseñen propuestas iSTEAM con objetivos educativos más amplios. 
Esta problemática, sin embargo, no es exclusiva de la iSTEAM. Aunque el desarrollo de secuencias de enseñanza-aprendizaje (SEA) basadas en resultados de investigación es uno de los propósitos del campo de la Didáctica de las Ciencias Experimentales, hasta el momento son escasos los marcos teóricos focalizados en los factores y procesos involucrados en su diseño (Arriassecq, Greca y Cayul 2017, Duit 2006, Méheut y Psillos 2004). Dentro de los modelos propuestos aparecen el abordaje de resolución de problemas, la reconstrucción educacional y la ingeniería didáctica (ID), basada en la Teoría de las Situaciones Didácticas de Brousseau (Artigue 1988), que plantea un análisis a priori para definir los problemas que deberá abordar el alumnado, teniendo en cuenta la dimensión epistemológica del contenido, la dimensión psicocognitiva y la dimensión didáctica.

Junto con la escasez de modelos también es reducida la literatura que defina explícitamente metodologías para la evaluación de las SEA y que brinde información detallada y pertinente a los docentes que pretendan aplicarlas en los diversos contextos educativos. Así, aunque existen propuestas que vinculan marcos teóricos con diseños e implementaciones, varias de ellas son, o bien utilizadas solo por quienes las han propuesto, o bien por un escaso número de investigadores (Guisasola, Zuza y Sagastibeltza 2019, Juuti y Lavonen 2006).

La necesidad de avanzar en el conocimiento sobre las características de las SEA y la validez de su aplicación en contextos más amplios ha llevado al desarrollo del enfoque metodológico denominado Investigación basada en el diseño (IBD), definido como «el estudio sistemático del diseño, el desarrollo y la evaluación de intervenciones educativas» (Plomp 2013 p. 11); por ejemplo, de programas, ambientes de aprendizaje o estrategias de enseñanza-aprendizaje. Como herramienta de diseño, la IBD constituye un marco lo suficientemente amplio y adaptable a diferentes propuestas específicas (Guisasola, Zuza, Ametller y GutiérrezBerraondo 2017), teniendo aspectos en común con otras metodologías de diseño de las SEA. Así, la IBD ha sido considerada como una generalización de la ID (Godino et al. 2013) que recurre a diferentes marcos teóricos como fundamentos para realizar investigaciones educativas. La IBD puede considerarse una familia de abordajes de investigación educativa circunscriptos por el interés en el diseño, la implementación y la evaluación de intervenciones educativas en contextos reales y específicos. Como herramienta de evaluación, la IBD hace uso de diversas herramientas metodológicas, tanto cualitativas como cuantitativas. Algunas fases comunes de los estudios de desarrollo basados en este paradigma metodológico son (Plomp 2013): la investigación preliminar —en la que se detectan las necesidades del contexto, se revisa la literatura y se construye el marco teórico-; la creación y revisión de prototipos en la que se diseñan y se ponen a prueba SEA revisadas en sucesivas iteraciones para refinar la intervención-; y la evaluación —en la que se concluye si la intervención cumple con las especificaciones predeterminadas-.

En este contexto, y volviendo al enfoque iSTEAM, su viabilidad pasa por la evaluación de diferentes modelos teóricos en escenarios educativos. Por ello, es relevante diseñar, implementar y evaluar diversas SEA para validar tanto estas - para que puedan ser usadas por docentes en sus entornos específicos- como modelos teóricos lo suficientemente concretos para que los docentes puedan adaptar y diseñar nuevas SEA. Así, dentro de una investigación más amplia, hemos desarrollado un modelo teórico para la iSTEAM siguiendo la línea francesa de la ID en un sentido amplio y diseñado, implementado y evaluado una SEA dentro del enfoque metodológico IBD - con el objetivo de potenciar el desarrollo competencial integral del alumnado de Educación Primaria (Ortiz-Revilla 2020). En este artículo, dado el espacio disponible, abordamos dos aspectos que consideramos centrales: la concreción del modelo teórico en el diseño de la SEA y su evaluación en términos del desarrollo de la competencia matemática y competencias básicas en ciencia y tecnología 
(CMCT) — más conocida como competencia científica — alcanzado por el alumnado mediante su implementación.

\section{Modelo teórico iSTEAM y su concreción en el diseño, implementación y la evaluación de la SEA}

En esta sección se esboza el modelo teórico para la iSTEAM (Ortiz-Revilla, Greca y Arriassecq 2018), detallando la información relevante para comprender el proceso de diseño, implementación y evaluación de la SEA.

Para la composición del modelo se parte de la postura epistemológica de Larry Laudan, para quien el progreso científico viene determinado por la cantidad de problemas que una teoría es capaz de resolver. Para ello, Laudan (1984) presenta la Red Triádica, un modelo que postula un análisis epistemológico del desarrollo científico compuesto por tres niveles de compromiso científico con el mismo estatus, que interactúan de manera compleja y cuyas modificaciones no siempre son simultáneas: el compromiso con las teorías, con los métodos y con los fines estos últimos también denominados objetivos o metas-. Son precisamente estos tres niveles de compromiso los que se adoptan para la composición de un modelo teórico cohesionado y coherente que da sustento a la iSTEAM. En el marco de la red triádica creada para justificar la iSTEAM, el fin consiste en potenciar el desarrollo competencial integral del alumnado. Para ello, resulta necesario el empleo de metodologías activas; en este modelo, se emplean la metodología de indagación y el diseño de ingeniería, que permiten desarrollar no solamente la competencia científica en su visión más comprensiva, sino también el resto de las competencias (Aguilera Morales et al. 2018, Kang 2019, Ortiz-Revilla, Greca y Adúriz-Bravo 2018). El diseño de ingeniería, en particular, es considerado en la literatura como una metodología clave para la integración de las diversas disciplinas STEAM. Por último, se incorpora a la red el nivel teórico, cuyos constructos, basados en la ID, se sustentan sobre tres ejes compatibles: epistemológico, psicológico y didáctico. Dentro del primero, esencial para la comprensión de los principios, fundamentos y métodos científicos, se adopta la postura de Laudan (1977). Para el segundo, la Teoría de los Campos Conceptuales de Vergnaud (1990), para quien el conocimiento se organiza en campos conceptuales - grandes conjuntos informales y heterogéneos de situaciones y problemas cuyo análisis y tratamiento requieren diferentes clases de conceptos, procedimientos y representaciones simbólicas, interrelacionadas - que el sujeto se apropia a lo largo del tiempo. Así, Vergnaud considera que un concepto adquiere paulatinamente significado para un sujeto, solamente si este se enfrenta a situaciones y problemas variados en los que dicho concepto esté implícita o explícitamente presente, pues de tales situaciones y problemas el sujeto puede abstraer las propiedades para conformar los esquemas mentales que den significado al concepto. Para el tercero, la noción de objetivo-obstáculo de Martinand (1986), quien propone la existencia de una relación dialéctica entre los objetivos de la enseñanza y los obstáculos que se interponen en el camino para lograrlos, que sirve de guía para el diseño de la SEA.

Este modelo fue aplicado en el diseño, implementación y evaluación de una SEA iSTEAM de 17 actividades agrupadas en 16 sesiones - esquematizada en la figura 1 - para sexto curso de Educación Primaria que abordó contenidos de Ciencias de la Naturaleza, Educación Plástica y Matemáticas —asignaturas presentes en el currículo español— de forma integrada a partir de la metodología de indagación guiada (Martin-Hansen 2002) y del diseño de ingeniería. Partiendo de la visión epistemológica laudaniana, la SEA se planteó en torno a la solución de un problema principal: ¿Cómo diseñar el mejor sistema de iluminación para mi sala de estudio? y los contenidos curriculares fueron abordados de manera interdisciplinaria a medida que el alumnado los necesitaba para alcanzar posibles soluciones. Así, se abordaron contenidos 
relacionados con la electricidad, como la electricidad estática, circuitos, materiales aislantes y conductores, transformaciones energéticas, etc. —Ciencias-; se usaron las TIC y se trataron aspectos tecnológicos ligados a la iluminación y sus avances - Tecnología—; se trabajó sobre el diseño de un sistema de iluminación de una habitación concreta —-Ingeniería-; se abordaron contenidos relacionados con el color y sus sensaciones - Artes—; y la información necesaria para decidir las bombillas a usar — considerándose el coste, la eficiencia energética y la vida útil, entre otros - fue tratada mediante funciones, tablas y gráficos - Matemáticas-. Todo ello con el fin de potenciar el desarrollo de las siete competencias clave propuestas dentro del Sistema Educativo Español en términos de los contenidos curriculares abordados para la resolución del problema principal.

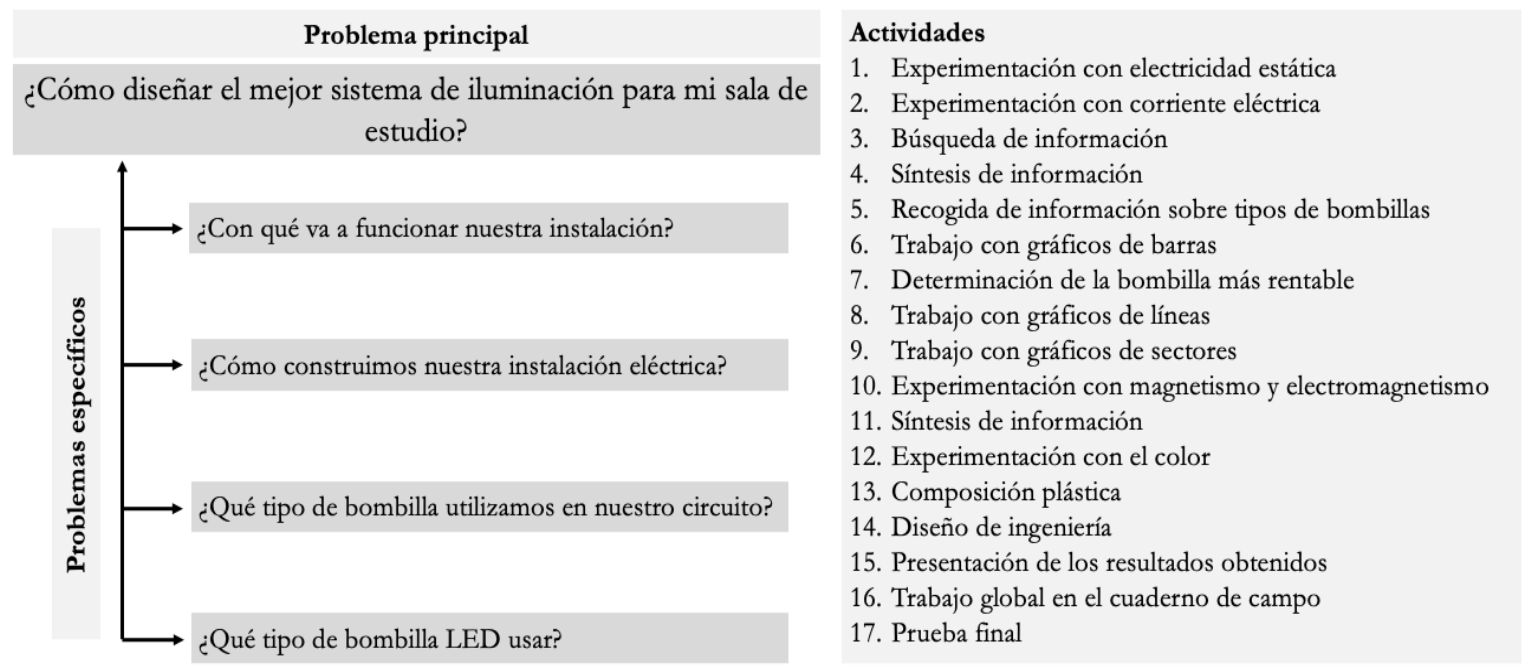

Figura 1. Esquema de la SEA iTEAM cuyo diseño completo se puede consultar en Ortiz-Revilla (2018).

Acercándonos al plano psicológico y didáctico, el proceso de diseño, implementación y evaluación comenzó por averiguar las representaciones — desde el punto de vista de Martinand - que poseía el alumnado sobre los contenidos abordados en la SEA; por ejemplo, sobre la electricidad, el tratamiento de datos matemáticos o el color. Este proceso se realizó mediante una consulta de la información presente en la literatura especializada ${ }^{1}$. Se decidió hacerlo así para evitar usar un instrumento antes de la intervención, buscando la menor interferencia posible en el desarrollo habitual de la actividad académica. Después, se seleccionaron algunas representaciones que no se correspondían con lo científicamente consensuado y que eran abordables en el limitado período de tiempo en el que se desarrolló la SEA. En línea con Astolfi (1999), el criterio para seleccionar las representaciones a abordar se determinó por el valor que su fisura suponía para el desarrollo competencial. Con las representaciones seleccionadas, se generaron los objetivos-representación, que guiaron el planteamiento de situaciones — desde la visión de Vergnaud - encaminadas a la fisura de dichas representaciones. Así, estos objetivos-representación, aunque lógicamente relacionados con los contenidos curriculares, se construyeron teniendo en cuenta las representaciones presentes en los esquemas mentales del alumnado, además de estar enfocados al problema principal planteado. Así, el proceso de enseñanza-aprendizaje se direccionó hacia el abordaje del obstáculo, en la búsqueda de la superación de aquellos construidos en la vida cotidiana (Bachelard 1938).

Cabe destacar que desde la iSTEAM viabilizada mediante las metodologías activas adoptadas, cada problema puede ofrecer una buena variedad de situaciones para el alumnado, por

\footnotetext{
${ }^{1}$ Este proceso también se puede realizar mediante la aplicación de test, mapas conceptuales, esquemas, historias, relatos, dibujos y un largo etcétera de métodos que dan pie a la detección de las representaciones o ideas alternativas del alumnado.
} 
ejemplo, para cada hipótesis trabajada. Así, cada indagación o diseño planteado implica una miríada de situaciones vergnaudianas desde el punto de vista del alumnado, permitiéndole abstraer propiedades y usar diferentes representaciones, que enriquecerán sus esquemas mentales y favorecerán la significación de los conceptos abordados (Greca y Ataíde 2019). Por ello, todo el proceso se operativizó dividiendo la SEA en cuatro problemas más específicos ¿con qué va a funcionar nuestra instalación?, ¿cómo construimos nuestra instalación eléctrica?, ¿qué tipo de bombilla utilizamos en nuestro circuito? y iqué tipo de bombilla led usar? - abordados a través de las fases características de la indagación guiada. Con el conocimiento adquirido en estas indagaciones, el alumnado estaba en condiciones, finalmente, de resolver el problema principal mediante el diseño de ingeniería.

En la figura 2 se detalla el modelo teórico desarrollado para la iSTEAM y su concreción en la SEA, con base en la red triádica.

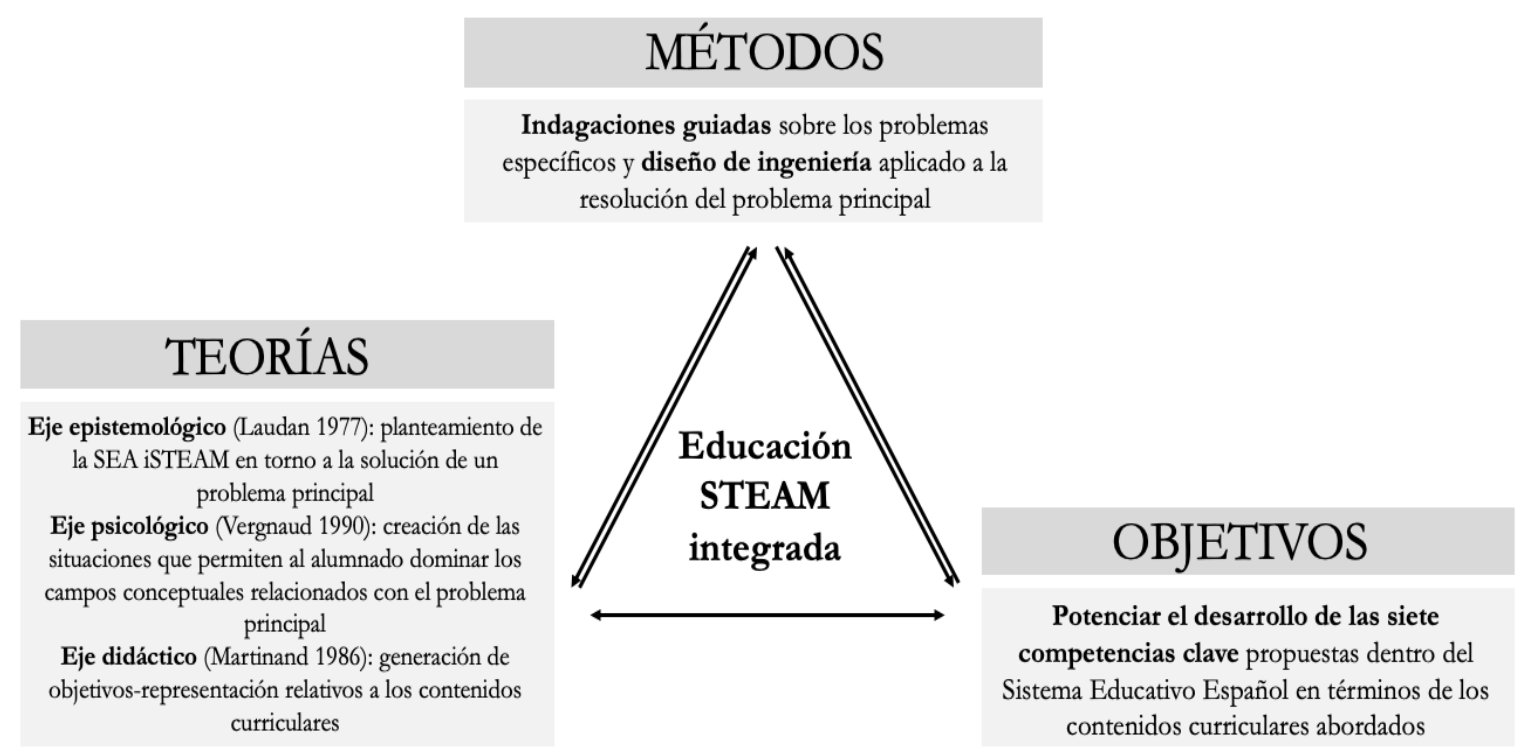

Figura 2. Esquema de funcionamiento triádico del modelo teórico de la iSTEAM y su concreción en la SEA. Adaptada de Ortiz-Revilla, Greca y Arriassecq (2018).

Siguiendo el enfoque IBD, la SEA fue evaluada a partir del desarrollo competencial alcanzado por el alumnado en base a la consecución de los estándares de aprendizaje evaluables (EAE) correspondientes a los contenidos abordados. La evaluación, realizada de forma conjunta por los docentes que implementaron la SEA y por el investigador, fue realizada después de la implementación de cada prototipo, lo que llevó a sucesivas modificaciones de la SEA original posibilitando una mejor consecución de los objetivos propuestos.

En la tabla 1 se muestra este proceso en relación con las tres asignaturas y los contenidos centrales abordados en la SEA para el desarrollo de la CMCT. 
Tabla 1. Diseño de la SEA en cuanto a los contenidos centrales para el desarrollo de la CMCT

\begin{tabular}{|c|c|c|c|c|c|}
\hline $\begin{array}{l}\text { A } \\
\text { s } \\
\text { i } \\
\text { g } \\
\text { } \\
\text { a } \\
\text { t } \\
\text { u } \\
\text { r } \\
\text { a }\end{array}$ & $\begin{array}{c}\text { Bloque/Contenido } \\
\text { abordado }\end{array}$ & EAE & $\begin{array}{l}\text { Representación } \\
\text { del alumnado } \\
\text {-idea previa- }\end{array}$ & $\begin{array}{l}\text { Objetivo- } \\
\text { representación } \\
\text { de la SEA }\end{array}$ & $\begin{array}{l}\text { Situaciones } \\
\text { propuestas }\end{array}$ \\
\hline $\begin{array}{l}\mathrm{N} \\
\mathrm{a} \\
\mathrm{t} \\
\mathrm{u} \\
\mathrm{r} \\
\mathrm{a} \\
\mathrm{l} \\
\mathrm{e} \\
\mathrm{z} \\
\mathrm{a}\end{array}$ & $\begin{array}{l}\text { Materia y Energía/La } \\
\text { luz como fuente de } \\
\text { energía. Electricidad: la } \\
\text { corriente eléctrica. } \\
\text { Circuitos eléctricos } \\
\text { La tecnología, objetos } \\
\text { y máquinas/La } \\
\text { electricidad en el } \\
\text { desarrollo de las } \\
\text { máquinas. Elementos } \\
\text { de los circuitos } \\
\text { eléctricos. Efectos de } \\
\text { la electricidad. } \\
\text { Conductores y } \\
\text { aislantes }\end{array}$ & $\begin{array}{l}\text { Conoce las leyes básicas que } \\
\text { rigen la transmisión de la } \\
\text { corriente eléctrica } \\
\text { Observa, identifica y explica } \\
\text { algunos efectos de la } \\
\text { electricidad } \\
\text { Expone ejemplos de materiales } \\
\text { conductores y aislantes, } \\
\text { argumentando su exposición } \\
\text { Observa e identifica los } \\
\text { elementos de un circuito } \\
\text { eléctrico aplicándolos para } \\
\text { construir uno }\end{array}$ & $\begin{array}{l}\text { Una corriente eléctrica } \\
\text { es una sustancia, } \\
\text { generalmente un fluido, } \\
\text { que se mueve a través } \\
\text { de cables } \\
\text { La batería es la única } \\
\text { fuente de cargas que se } \\
\text { inyecta en los cables } \\
\text { como el agua de una } \\
\text { tubería } \\
\text { La corriente } \\
\text { suministrada por la } \\
\text { batería se consume a lo } \\
\text { largo del circuito } \\
\text { En un circuito simple, } \\
\text { la conexión a un solo } \\
\text { terminar de la batería } \\
\text { es suficiente para } \\
\text { encender las bombillas }\end{array}$ & $\begin{array}{l}\text { Conocer la } \\
\text { composición } \\
\text { química de las } \\
\text { baterías } \\
\text { Entender la } \\
\text { función de los } \\
\text { electrones en la } \\
\text { corriente eléctrica } \\
\text { Comprender el } \\
\text { funcionamiento de } \\
\text { un circuito }\end{array}$ & $\begin{array}{l}\text { Sesiones } 4 \text { y } 5 \\
\text { Indagación guiada } \\
\text { ¿cómo } \\
\text { construimos } \\
\text { nuestra instalación } \\
\text { eléctrica? Hipótesis } \\
\text { relacionadas con } \\
\text { circuitos, } \\
\text { materiales } \\
\text { conductores y } \\
\text { aislantes y circuitos } \\
\text { en serie y paralelo }\end{array}$ \\
\hline $\begin{array}{l}\mathrm{M} \\
\mathrm{a} \\
\mathrm{t} \\
\mathrm{e} \\
\mathrm{m} \\
\mathrm{a} \\
\mathrm{t} \\
\mathrm{i} \\
\mathrm{c} \\
\mathrm{a} \\
\mathrm{s}\end{array}$ & $\begin{array}{l}\text { Estadística y } \\
\text { probabilidad/Tratamie } \\
\text { nto de la información }\end{array}$ & $\begin{array}{l}\text { Interpreta datos, realiza tablas y } \\
\text { utiliza diferentes gráficos para } \\
\text { su representación, con la } \\
\text { información obtenida en su } \\
\text { entorno } \\
\text { Recoge y clasifica datos } \\
\text { cuantitativos, de situaciones de } \\
\text { su entorno, utilizándolos para } \\
\text { construir tablas de frecuencias } \\
\text { absolutas y relativas } \\
\text { Realiza análisis crítico y } \\
\text { argumentado sobre las } \\
\text { informaciones que se presentan } \\
\text { mediante gráficos estadísticos } \\
\text { Resuelve problemas que } \\
\text { impliquen dominio de los } \\
\text { contenidos propios de } \\
\text { estadística, utilizando estrategias } \\
\text { heurísticas, de razonamiento, } \\
\text { creando conjeturas, } \\
\text { construyendo, argumentando, } \\
\text { tomando y valorando decisiones }\end{array}$ & $\begin{array}{l}\text { Dificultad para } \\
\text { contextualizar datos en } \\
\text { relación con un } \\
\text { contexto real } \\
\text { La reorganización de } \\
\text { los datos en bruto } \\
\text { resulta "tortuosa" } \\
\text { Todos los tipos de } \\
\text { gráficos sirven para } \\
\text { representar cualquier } \\
\text { dato }\end{array}$ & $\begin{array}{l}\text { Recoger, clasificar, } \\
\text { representar e } \\
\text { interpretar datos } \\
\text { obtenidos en un } \\
\text { contexto real }\end{array}$ & $\begin{array}{l}\text { Sesiones } 6,7,8,9 \text { y } \\
\text { 10: indagación } \\
\text { guiada ¿qué tipo de } \\
\text { bombilla } \\
\text { utilizamos en } \\
\text { nuestro circuito? } \\
\text { Hipótesis } \\
\text { relacionadas con el } \\
\text { coste, la eficiencia } \\
\text { energética y la vida } \\
\text { útil de distintos } \\
\text { tipos de bombillas, } \\
\text { tratadas mediante } \\
\text { funciones, tablas y } \\
\text { gráficos. }\end{array}$ \\
\hline
\end{tabular}


Tabla 1. Continuación

\begin{tabular}{|c|c|c|c|c|c|}
\hline $\begin{array}{l}\mathrm{i} \\
\mathrm{g} \\
\mathrm{n} \\
\mathrm{a} \\
\mathrm{t} \\
\mathrm{u}\end{array}$ & $\begin{array}{c}\text { Bloque/Contenido } \\
\text { abordado }\end{array}$ & EAE & $\begin{array}{l}\text { Representación } \\
\text { del alumnado } \\
\text {-idea previa- }\end{array}$ & $\begin{array}{l}\text { Objetivo- } \\
\text { representación } \\
\text { de la SEA }\end{array}$ & $\begin{array}{l}\text { Situaciones } \\
\text { propuestas }\end{array}$ \\
\hline $\begin{array}{l}\mathrm{u} \\
\mathrm{c} \\
\mathrm{a} \\
\mathrm{c} \\
\mathrm{i} \\
\mathrm{O} \\
\mathrm{n}\end{array}$ & $\begin{array}{l}\text { Expresión } \\
\text { artística/El dibujo } \\
\text { de representación y } \\
\text { la composición } \\
\text { plástica y visual }\end{array}$ & $\begin{array}{l}\text { Conoce la simbología de los } \\
\text { colores fríos y cálidos y } \\
\text { aplica dichos conocimientos } \\
\text { para transmitir diferentes } \\
\text { sensaciones en las } \\
\text { composiciones plásticas que } \\
\text { realiza }\end{array}$ & $\begin{array}{l}\text { El color percibido } \\
\text { por el observador es } \\
\text { siempre el del } \\
\text { cuerpo (el color es } \\
\text { una propiedad } \\
\text { intrínseca de la } \\
\text { materia) } \\
\text { El color percibido } \\
\text { por el observador es } \\
\text { el color con el que } \\
\text { la bombilla ilumina } \\
\text { el cuerpo (el color } \\
\text { es una propiedad } \\
\text { del iluminador) } \\
\text { El color percibido } \\
\text { por el observador es } \\
\text { la suma del color de } \\
\text { la bombilla y el } \\
\text { color del cuerpo } \\
\text { (ambos "emiten" su } \\
\text { propio color, que el } \\
\text { ojo luego suma) } \\
\text { El color se explica } \\
\text { por los pigmentos } \\
\text { (luz y color son } \\
\text { entidades físicas } \\
\text { independientes) }\end{array}$ & $\begin{array}{l}\text { Conocer los } \\
\text { factores que } \\
\text { influyen en la } \\
\text { percepción } \\
\text { visual del color } \\
\text { de un cuerpo } \\
\text { Aplicar el } \\
\text { conocimiento } \\
\text { sobre la } \\
\text { percepción del } \\
\text { color para } \\
\text { realizar } \\
\text { composiciones } \\
\text { plásticas }\end{array}$ & $\begin{array}{l}\text { Sesiones } 11 \text { y } \\
\text { 12: Indagación } \\
\text { guiada ¿Qué } \\
\text { tipo de bombilla } \\
\text { led usar? } \\
\text { Hipótesis } \\
\text { relacionadas con } \\
\text { la percepción } \\
\text { del color y } \\
\text { composición } \\
\text { plástica con } \\
\text { colores cálidos y } \\
\text { fríos }\end{array}$ \\
\hline
\end{tabular}

Cabe destacar que, además de los EAE aquí recogidos, forman parte del desarrollo de la CMCT — en términos de las dimensiones competenciales propuestas por Ortiz-Revilla, Greca y Adúriz-Bravo (2021) - aquellos EAE derivados de algunos contenidos del Bloque 1 Iniciación a la actividad científica de la asignatura de Ciencias de la Naturaleza —-Iniciación a la actividad científica. Aproximación experimental a algunas cuestiones relacionadas con las Ciencias de la Naturaleza; Utilización de diferentes fuentes de información. Observación directa e indirecta de la naturaleza empleando instrumentos apropiados y a través del uso de libros, medios audiovisuales y tecnológicos; Trabajo individual y en grupo; Planificación de proyectos y presentación de informes-, del Bloque 1 Procesos, métodos y actitudes en Matemáticas de la asignatura de Matemáticas —Planificación del proceso de resolución de problemas: Análisis y comprensión del enunciado. Estrategias y procedimientos: gráficos, tablas, esquemas de la situación, datos, planteamiento, ensayo y error razonado, selección de las operaciones, etc. Resultados obtenidos y valoración de los mismos; Disposición para desarrollar aprendizajes autónomos y confianza en sus propias capacidades para desarrollar actitudes adecuadas y afrontar las dificultades propias del trabajo científico; Interés y curiosidad por el aprendizaje y utilización de las Matemáticas-y del Bloque 1 Educación 
audiovisual de la asignatura de Educación Plástica — Las TIC para el tratamiento de imágenes, diseño y animación, y su empleo para la difusión de los trabajos elaborados; Uso de programas digitales de edición y procesado de imagen, video y texto-.

\section{Preguntas de investigación}

La evaluación de la SEA y, por tanto, del modelo teórico subyacente, ha sido guiada por las siguientes preguntas de investigación:

- ¿Cómo se modifica la SEA iSTEAM a lo largo de las sucesivas implementaciones para potenciar el desarrollo competencial del alumnado?

- ¿Qué efecto produce la implementación de la SEA iSTEAM en el desarrollo de la CMCT del alumnado?

\section{Metodología}

\section{Diseño de la evaluación}

Se combinaron y complementaron los enfoques cualitativo y cuantitativo, estableciéndose una investigación de métodos mixtos (Creswell y Creswell 2018). Siguiendo las fases de la IBD, se implementaron iteraciones de tres prototipos de la SEA iSTEAM — con una duración aproximada de seis semanas cada una- en seis grupos. El Prototipo 1 se ejecutó en un solo grupo, el Prototipo 2 en dos grupos y el Prototipo 3 en tres grupos. La ampliación de la muestra de alumnado fue progresiva desde la implementación del primer prototipo producto del investigador- hacia los sucesivos prototipos — productos de la reflexión conjunta, basada en las evidencias disponibles, del investigador y los docentes.

\section{Contexto y participantes}

La SEA iSTEAM fue implementada durante el curso académico 2017-2018 —el Prototipo 1 en las tres primeras semanas de diciembre de 2017, el Prototipo 2 en las tres primeras semanas de febrero de 2018 y el Prototipo 3 en las dos últimas semanas abril y la primera de mayo de 2018 - en el Colegio de Educación Infantil y Primaria (CEIP) público Fernando de Rojas, situado en la ciudad de Burgos - España- La temporalización fue acordada con todos los docentes para posibilitar la revisión y mejora de los sucesivos prototipos a partir del análisis de cada implementación. Cabe destacar que las 16 sesiones se llevaron a cabo en las horas de las asignaturas de Ciencias de la Naturaleza, Matemáticas y Educación Plástica, de forma independiente al contenido especifico abordado en las sesiones. Dado que estas asignaturas eran impartidas en todos los casos por el tutor, solamente intervino un docente en cada grupo.

En el estudio participaron un total de 121 escolares - 54.54\% chicos_-, divididos en seis grupos $-n=20, n=21, n=20, n=20, n=19$ y $n=21$, respectivamente- con sus respectivos docentes y con edades entre los 11 y los 13 años $-M=11.33, D E=.52$. Pertenecían en su mayoría a la clase obrera y existía un cierto porcentaje de alumnado migrante de variedad de nacionalidades. Todos ellos cursaban el sexto curso de Educación Primaria y no hubo casos perdidos.

Los docentes fueron los tutores de los grupos, contándose con cinco maestros generalistas dos de ellos con formación en ciencias naturales y nuevas tecnologías- y una especialista en inglés. El maestro de la primera iteración fue el que contó con más acompañamiento en la práctica, debido a que al aumentar el número de grupos en las sucesivas iteraciones el investigador tuvo que dividir su tiempo de estancia en cada aula. Habitualmente, la asignatura Ciencias de la Naturaleza se impartía de manera tradicional, basada en exposiciones y libro de texto. 
El esquema de ampliación de la muestra en base a la secuenciación temporal de las tres iteraciones con sus respectivos prototipos se ilustra en la figura 3.

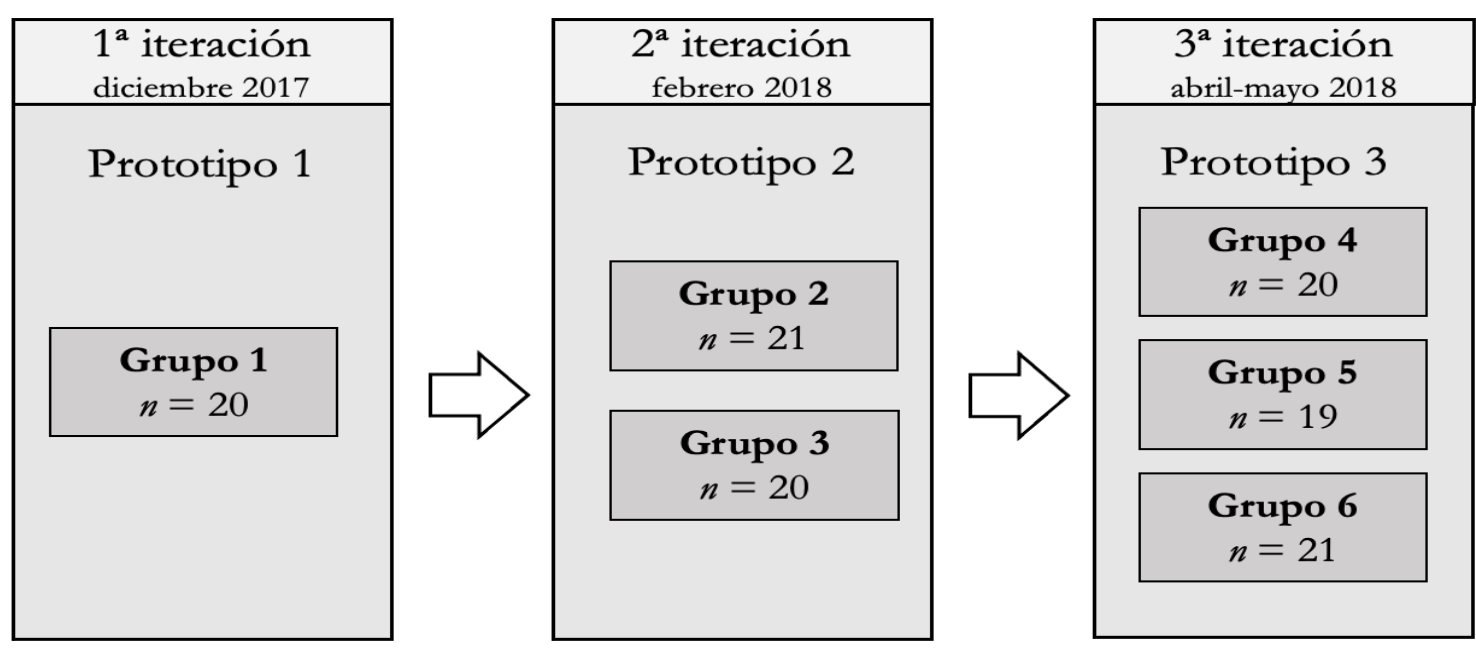

Figura 3. Esquema de ampliación de la muestra en base a la secuenciación temporal de las tres iteraciones sus respectivos prototipos. Adaptada de Ortiz-Revilla (2020).

La estrategia de selección de la muestra se correspondió con un muestreo no probabilístico por conveniencia, eligiéndose el centro para contar con seis líneas para el desarrollo de las tres iteraciones programadas.

\section{Recolección de datos}

Como técnicas de recolección de datos, desde la parte cualitativa se empleó la observación participante a través del registro de notas de campo (Spradley 2016) por parte del investigador y de los maestros inmersos en el contexto de enseñanza-aprendizaje y el material elaborado por el alumnado (Massot Lafon, Dorio Alcaraz y Sabariego Puig 2004), en este caso su cuaderno de campo personal. Desde la parte cuantitativa se empleó un conjunto de datos numéricos emergentes del análisis cualitativo.

\section{Análisis de datos}

En las dos semanas siguientes a cada iteración, las notas de campo de la observación participante y un análisis rápido de los cuadernos de campo del alumnado fueron objeto de una interpretación conjunta entre el investigador y los docentes implicados en dicha iteración, teniendo en consideración el alcance de los objetivos-representación, algunos de los cuales aparecen en la tabla 1. La información se redujo y se organizó en cinco categorías por razonamiento inductivo, siguiendo la lógica de la estructuración de la SEA: modificaciones generales, modificaciones relativas al área de Matemáticas, modificaciones relativas al área de Ciencias de la Naturaleza, modificaciones relativas al área de Educación Plástica y modificaciones relativas al diseño de ingeniería. Las opiniones, las sugerencias de mejora y los conocimientos del investigador y de los seis maestros participantes fueron empleadas para volver a diseñar los sucesivos prototipos, prestándose máxima atención a las necesidades contextuales.

Por otra parte, para poder determinar la influencia de la implementación de la SEA iSTEAM en el desarrollo competencial se siguieron las directrices de Ortiz-Revilla y Greca (2019) a través de un análisis documental (del Rincón Igea, Arnal Agustín, Latorre Beltrán y Sans Martín 1995) de los cuadernos de campo del alumnado. Para ello, cada maestro calificó, según su propio criterio, las 17 actividades de la SEA en bien, regular o mal. A través de una relación de las actividades con la consecución de los EAE y de estos con su contribución al desarrollo 
de cada competencia clave, se pudo calcular el porcentaje de consecución de cada competencia clave con relación a su valor máximo.

Por último, se comparó el desarrollo de la CMCT —en función de sus respectivos EAE— del alumnado al inicio —experimentación con la electricidad estática - y próximo al final experimentación con magnetismo y electromagnetismo - de la SEA, que conformaron las variables CMCT inicial y CMCT final. Las actividades están relacionadas con el mismo EAE Realiza pequeñas investigaciones, planteando problemas, enunciando bipótesis, seleccionando el material necesario, extrayendo conclusiones y comunicando resultados y engloban la mayoría de las dimensiones de la CMCT. Dado que cada actividad puntuaba globalmente de forma algo diferente dependiendo del número de EAE a los cuales estaba asociada, para compararlas fueron transformadas a una misma escala (Fournier y Garnier 1990). Para esta comparación, se usó la versión 26.0 del software estadístico IBM SPSS Statistics. La prueba de Kolmogorov-Smirnov, con la corrección de Lilliefors, arrojó un p-valor $<.01$ significativo en las dos variables, lo que indicó una violación de la suposición de normalidad, empleándose así estadísticos no paramétricos.

\section{Resultados y discusión}

\section{Modificaciones de la SEA iSTEAM a lo largo de las sucesivas implementaciones}

La información obtenida de las notas de campo y de la evaluación competencial escolar, dio lugar a las modificaciones que fueron incorporadas a la programación del Prototipo 2 de la SEA iSTEAM, relacionadas con la organización de las actividades, la dificultad percibida en el alumnado para interpretar algunos fragmentos de la propuesta y la detección de carencias en el desarrollo de determinadas dimensiones competenciales, por ejemplo, la solvencia comunicativa. Los problemas encontrados, así como las principales modificaciones se muestran en la tabla 2.

Tabla 2. Problemas encontrados y principales modificaciones incorporadas a la programación del Prototipo 2 de la SEA iSTEAM.

\begin{tabular}{|c|c|}
\hline Problemas & Modificaciones \\
\hline $\begin{array}{l}\text { Generales: } \\
\text { - Falta de espacio en el } \\
\text { cuaderno de campo. } \\
\text { - Pérdidas de tiempo. } \\
\text { - Escasa solvencia } \\
\text { comunicativa. }\end{array}$ & $\begin{array}{l}\text { Ampliar la extensión del cuaderno de campo. } \\
\text { En lugar de comenzar a realizar la presentación de diapositivas como tarea para } \\
\text { hacer en casa, esta actividad se convierte en preparar un boceto preliminar. Así, } \\
\text { cada equipo construirá la presentación de diapositivas basándose en el boceto } \\
\text { preparado previamente. } \\
\text { En las fichas, indicar explícitamente que las respuestas sean fundamentadas y no se } \\
\text { limiten a palabras sueltas, haciendo hincapié, cuando se presenta la metodología de } \\
\text { la indagación en la formulación de hipótesis. } \\
\text { Los momentos dedicados a las ideas previas serán más breves debido a que cuando } \\
\text { se extienden, se tiende a repetir información y se demora tiempo innecesariamente. } \\
\text { Durante la explicación de la realización de las presentaciones comentar con el } \\
\text { alumnado que en el momento de la exposición no se puede limitar a leer } \\
\text { textualmente, sino que debe conformar un relato que demuestre poseer una idea } \\
\text { más amplia del tema que presenta. }\end{array}$ \\
\hline $\begin{array}{l}\text { Relativos al área de } \\
\text { Matemáticas: } \\
\text { - Dificultades técnicas en la } \\
\text { construcción de gráficos. } \\
\text { - Comprensión superficial de } \\
\text { la utilización e interpretación } \\
\text { de gráficos. }\end{array}$ & $\begin{array}{l}\text { Construir los gráficos de barras y poligonal en hojas cuadriculadas. Enfatizar en } \\
\text { que las marcas de graduación de los ejes deben estar siempre a la misma distancia. } \\
\text { Solicitar que el alumnado realice una pequeña interpretación en el problema del } \\
\text { gráfico poligonal. } \\
\text { Durante la realización de todos los tipos de gráficos discutir las funciones que } \\
\text { tiene cada tipo. } \\
\text { En los gráficos sectoriales incorporar la realización de una leyenda con colores. }\end{array}$ \\
\hline
\end{tabular}


Tabla 2. Continuación

\begin{tabular}{|l|l|}
\hline Problemas & Modificaciones \\
\hline $\begin{array}{l}\text { Relativos al área de Ciencias } \\
\text { de la Naturaleza: }\end{array}$ & $\begin{array}{l}\text { En la actividad de búsqueda de información en internet solicitar al alumnado } \\
\text { que las ideas que apunte en su cuaderno de campo sea capaz de explicarlas, para } \\
\text { - Dificultad para comprender } \\
\text { y sintetizar información } \\
\text { relevante. } \\
\text { - Dificultad en la } \\
\text { reorganización de datos. }\end{array}$ \\
$\begin{array}{l}\text { En vez de que el alumnado apunte las ideas a la vez que construye las } \\
\text { explicaciones científicas con el maestro, añadir como deber que realice un breve } \\
\text { resumen de cada explicación en un máximo de una cara, con el objetivo de } \\
\text { sintetizar la información más relevante, con pautas más claras por parte del } \\
\text { maestro. } \\
\text { En la actividad del recuento del tipo de bombillas que cada alumno tiene en su } \\
\text { casa, solicitar que se recojan los datos en una tabla. }\end{array}$ \\
\hline $\begin{array}{l}\text { Relativos al área de } \\
\text { Educación Plástica: } \\
\text { - Pérdidas de tiempo. } \\
\text { - Reflexión escasa. }\end{array}$ & $\begin{array}{l}\text { Reducir el número de experimentos de la ficha del color y agregar la cuestión } \\
\text { ipor qué? después de preguntar por el tipo de bombilla más adecuada para } \\
\text { iluminar la sala de estudio. }\end{array}$ \\
\hline $\begin{array}{l}\text { Relativas al diseño de } \\
\text { ingeniería: } \\
\text { - Dificultades técnicas de } \\
\text { diseño. } \\
\text { - Reflexión escasa. }\end{array}$ & $\begin{array}{l}\text { En el dibujo del sistema de iluminación, añadir la pauta de que esté } \\
\text { proporcionado, incorporando una escala. } \\
\text { Solicitar al alumnado una explicación final después del dibujo del sistema de } \\
\text { iluminación, justificando lo creado. }\end{array}$ \\
\hline
\end{tabular}

Tras la implementación del Prototipo 2 se volvió a realizar una interpretación conjunta de las notas de campo, esta vez llevaba a cabo entre el investigador y los tres docentes hasta ese momento implicados. Se consideró apropiado usar las mismas categorías que en la primera ocasión, que dieron lugar a un conjunto de modificaciones — mucho más reducido que en el caso anterior-incorporadas a la programación del Prototipo 3 de la SEA iSTEAM. En esta ocasión, las modificaciones ya no estuvieron relacionadas con la organización de las actividades — salvo una pequeña subsanación de temporalización — sino con cuestiones más específicas del enfoque de ciertas actividades y de autonomía de trabajo del alumnado. Los problemas encontrados, así como las principales modificaciones se muestran en la tabla 3.

Tabla 3. Problemas encontrados y principales modificaciones incorporadas a la programación del Prototipo 3 de la SEA iSTEAM.

\begin{tabular}{|l|l|}
\hline Problemas & Modificaciones \\
\hline $\begin{array}{l}\text { Relativos a contenidos de } \\
\text { - Visión reducida del desarrollo } \\
\text { tecnológico actual. } \\
\text { - Margen de mejora en la } \\
\text { construcción de gráficos. }\end{array}$ & $\begin{array}{l}\text { Después de la realización de los gráficos de sectores individual y grupal, } \\
\text { además de responder a la pregunta ¿qué conviene hacer en mi casa?, se } \\
\text { solicitará también respuesta a la cuestión ¿qué convendría hacer en la } \\
\text { clase en general? Así, se obtendrá una visión más global del camino hacia } \\
\text { el que se dirige el desarrollo tecnológico en las condiciones de vida actual. } \\
\text { Revisar con el alumnado lo que conoce acerca del diseño de los gráficos } \\
\text { de barras, incidiendo en los parámetros de disposición de las barras. }\end{array}$ \\
\hline $\begin{array}{l}\text { Relativos a contenidos de Ciencias } \\
\text { de la Naturaleza: } \\
\text { - Rigurosidad terminológica } \\
\text { mejorable. }\end{array}$ & $\begin{array}{l}\text { Ser más precisos en la terminología: en la ficha de los tipos de bombilla } \\
\text { cambiar consumo por consumo en una hora. }\end{array}$ \\
\hline $\begin{array}{l}\text { Relativos al diseño de ingeniería: } \\
\text { - Homogeneidad apreciable en los } \\
\text { diseños. }\end{array}$ & $\begin{array}{l}\text { Modificar la ficha de los requisitos del problema ingenieril para presentar } \\
\text { un problema menos guiado, con más opción a que el alumnado desarrolle } \\
\text { su creatividad. }\end{array}$ \\
\hline
\end{tabular}


Para ejemplificar cómo estas modificaciones incidieron en las competencias desarrolladas por el alumnado, la figura 4 muestra la mejora en las habilidades del alumnado en relación con una actividad concreta de funciones y gráficos a lo largo de los prototipos. Esta actividad - la número 6 de la figura 1 -, se relaciona con el objetivo-representación del área de Matemáticas de Recoger, clasificar, representar e interpretar datos obtenidos en un contexto real. Se puede observar que tras las primeras modificaciones introducidas que incentivaron el empleo de papel cuadriculado haciendo hincapié en que las marcas de graduación de los ejes debían colocarse siempre a la misma distancia, los gráficos de barras aparecen más cuidados. Tras las segundas modificaciones, que se centraron en la discusión de los parámetros de disposición de las barras, los gráficos muestran una notable mejoría también detectada en la interpretación del alumnado.
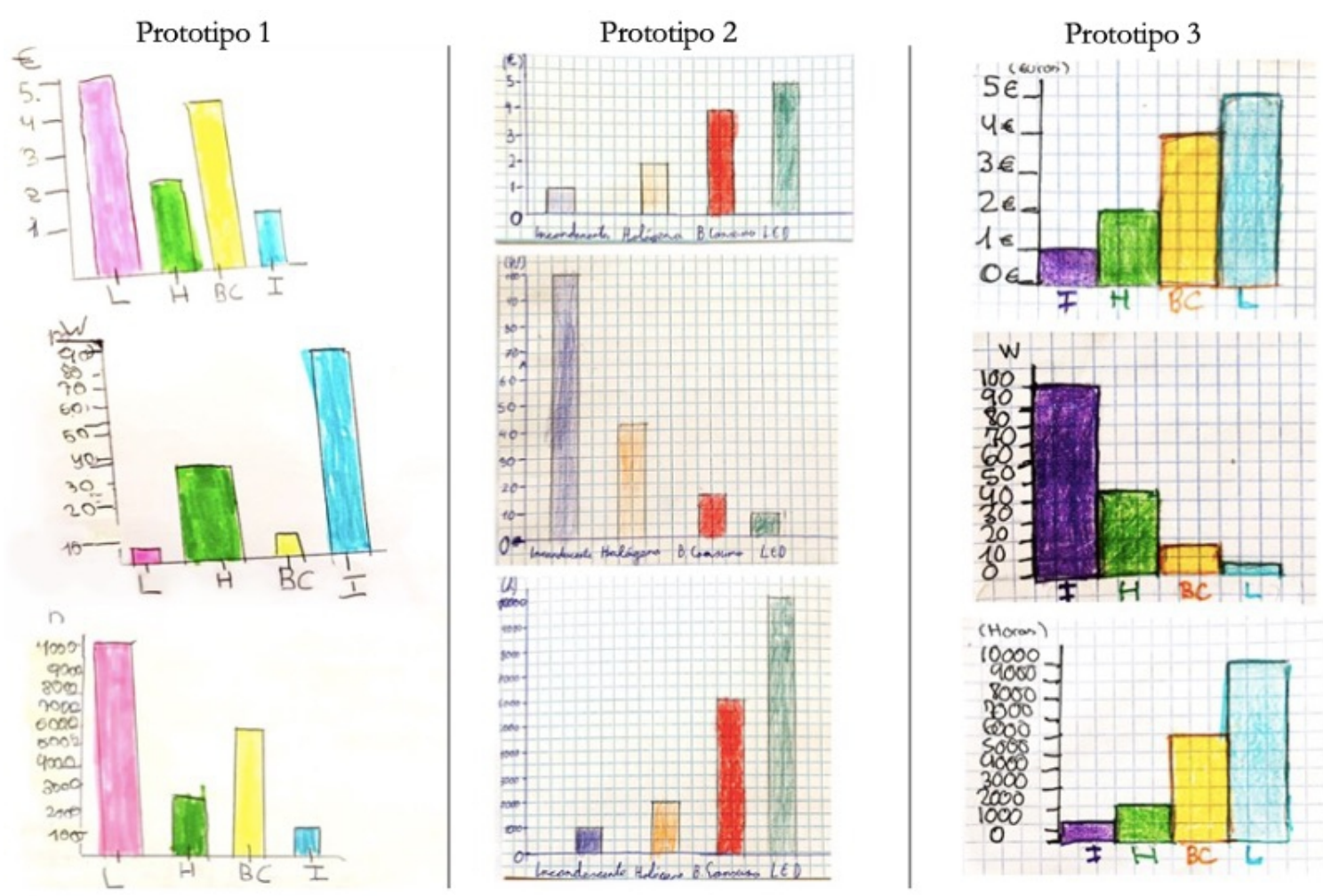

Figura 4. Ejemplificación de la mejora en el desarrollo de una actividad relacionada con contenidos de Matemáticas a raíz de las modificaciones introducidas a lo largo de los prototipos.

Los resultados presentados muestran como el proceso iterativo de aplicación y evaluación de la SEA, de manera conjunta con los docentes, ha permitido mejorar el primer prototipo adaptándose mejor al contexto educativo — conformado por el alumnado, los docentes y el centro escolar - y, con ello, mejorando el desempeño del alumnado en conexión con el rediseño de la SEA. Esto concuerda con los resultados de las investigaciones que usan IBD (entre otros, Barab y Squire 2004, Guisasola et al. 2017).

\section{Efectos de la implementación de la SEA iSTEAM en la CMCT del alumnado}

En cuanto al desarrollo específico de la $\mathrm{CMCT}^{2}$ por parte del alumnado se muestran, en primer lugar, los estadísticos descriptivos de su evaluación en cada prototipo — tabla 4 - y en el total de la muestra, aclarando que los puntajes a alcanzar en esta competencia podían variar de un mínimo de 0 a un máximo de 127.

\footnotetext{
2 Como se ha comentado, este estudio forma parte de una investigación más amplia; todos los resultados relativos al desarrollo competencial del alumnado en cada una de las competencias clave se pueden consultar en Ortiz-Revilla (2020).
} 
Tabla 4. Estadísticos descriptivos de CMCT por prototipos y en el total de la muestra.

\begin{tabular}{|l|c|c|c|c|}
\hline & Prototipo 1 & Prototipo 2 & Prototipo 3 & Total \\
\hline $\mathrm{n}(\mathrm{N})$ & 20 & 41 & 60 & $(121)$ \\
\hline $\mathrm{Me}$ & 106,2 & 112,5 & 117 & 115 \\
\hline$M$ & 100,2 & 107 & 111,8 & 108,3 \\
\hline $\mathrm{DE}$ & 24,4 & 18,4 & 15,4 & 18,5 \\
\hline $\begin{array}{l}\% \text { de consecución } \\
\text { competencial }\end{array}$ & 78,9 & 84,2 & 88 & 85,2 \\
\hline
\end{tabular}

Los estadísticos indican que tanto la media como, por tanto, el porcentaje de consecución de la CMCT, aumenta en cada una de las sucesivas iteraciones, siendo en todos los casos valores elevados para la mayor parte del alumnado, como se aprecia en la distribución de frecuencias por prototipos en la figura 5 .
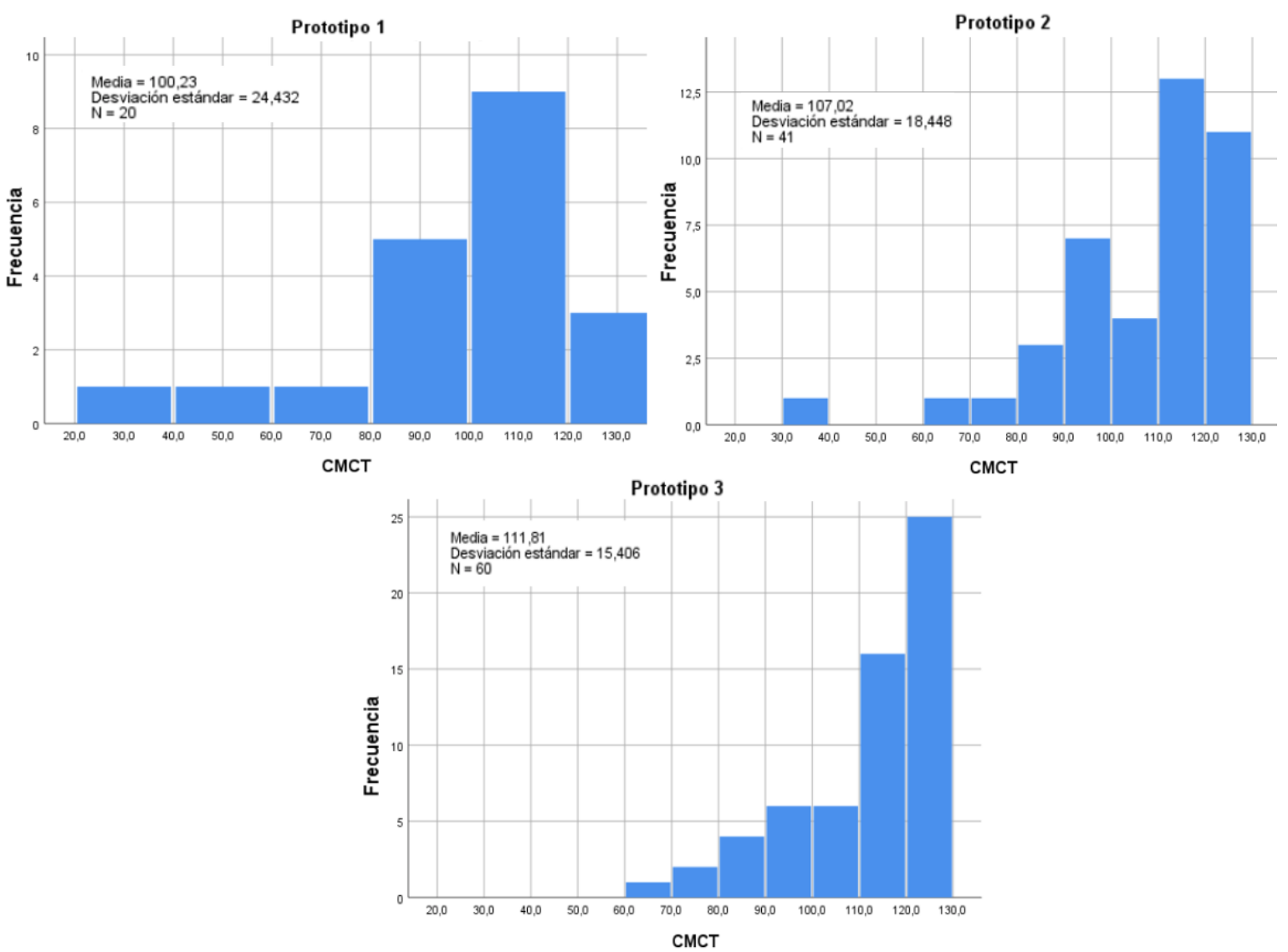

Figura 5. Distribución de frecuencias de los puntajes alcanzados en la CMCT en cada prototipo.

Comparando las variables CMCT inicial y CMCT final en el total de la muestra - cuyos estadísticos aparecen en la tabla 5-, se observa que los valores alcanzados son altos desde las primeras actividades, lo que contrasta con la manera tradicional de enseñanza en las clases, donde los aspectos del EAE indicado no eran trabajados, lo que sí se realizó explícitamente desde la primera actividad de la SEA. Aun así, la prueba de signos para muestras relacionadas reveló la existencia de diferencias significativas entre las medianas de los valores obtenidos por el alumnado en la CMCT inicial y final, con un p-valor de .015. Por tanto, existe evidencia de que la SEA iSTEAM implementada ayudó a mejorar el desarrollo de la CMCT, con un nivel de significancia del 5\%. 
Tabla 5. Estadísticos descriptivos de las variables CMCT inicial y final para el total de la muestra

\begin{tabular}{|l|c|c|}
\hline & CMCT inicial & CMCT final \\
\hline $\mathrm{N}$ & 121 & 121 \\
\hline$M e$ & 100,000 & 100,000 \\
\hline$M$ & 90,083 & 92,975 \\
\hline$D E$ & 17,224 & 21,704 \\
\hline
\end{tabular}

A continuación, se muestra un ejemplo del desarrollo competencial en un aspecto concreto de la CMCT, la formulación de hipótesis. La figura 6 ilustra el progreso de tres sujetos en el desarrollo de una actividad inicial y otra del tercio final, ambas que requieren de esta destreza.

Actividad inicial

\begin{tabular}{|c|c|c|}
\hline & EXPERIMENTO & $\begin{array}{c}\text { HIPÓTESIS: } \\
\text { ¿Qué crees que va a } \\
\text { ocurrir? }\end{array}$ \\
\hline 을 & $\begin{array}{l}\text { Interponer los } \\
\text { siguientes materiales } \\
\text { en el circuito: plástico, } \\
\text { hierro, madera, grafito, } \\
\text { cobre y papel. }\end{array}$ & $\begin{array}{l}\text { Plastico:no } \\
\text { Hietro: si } \\
\text { Madera: no } \\
\text { Grafito:no } \\
\text { Cobre:si } \\
\text { Papel.no }\end{array}$ \\
\hline
\end{tabular}

Actividad posterior

\begin{tabular}{|l|l|}
\hline \multicolumn{1}{|c|}{ EXPERIMIENTO } & $\begin{array}{c}\text { HIPÓTESIS: } \\
\text { ¿Qué crees que va } \\
\text { a ocurrir? }\end{array}$ \\
\hline $\begin{array}{l}\text { Acercar un imán a un } \\
\text { cable de cobre. }\end{array}$ & $\begin{array}{c}\text { Que va lo a } \\
\text { atraer al cobre } \\
\text { pero el plastico no. }\end{array}$ \\
\hline
\end{tabular}
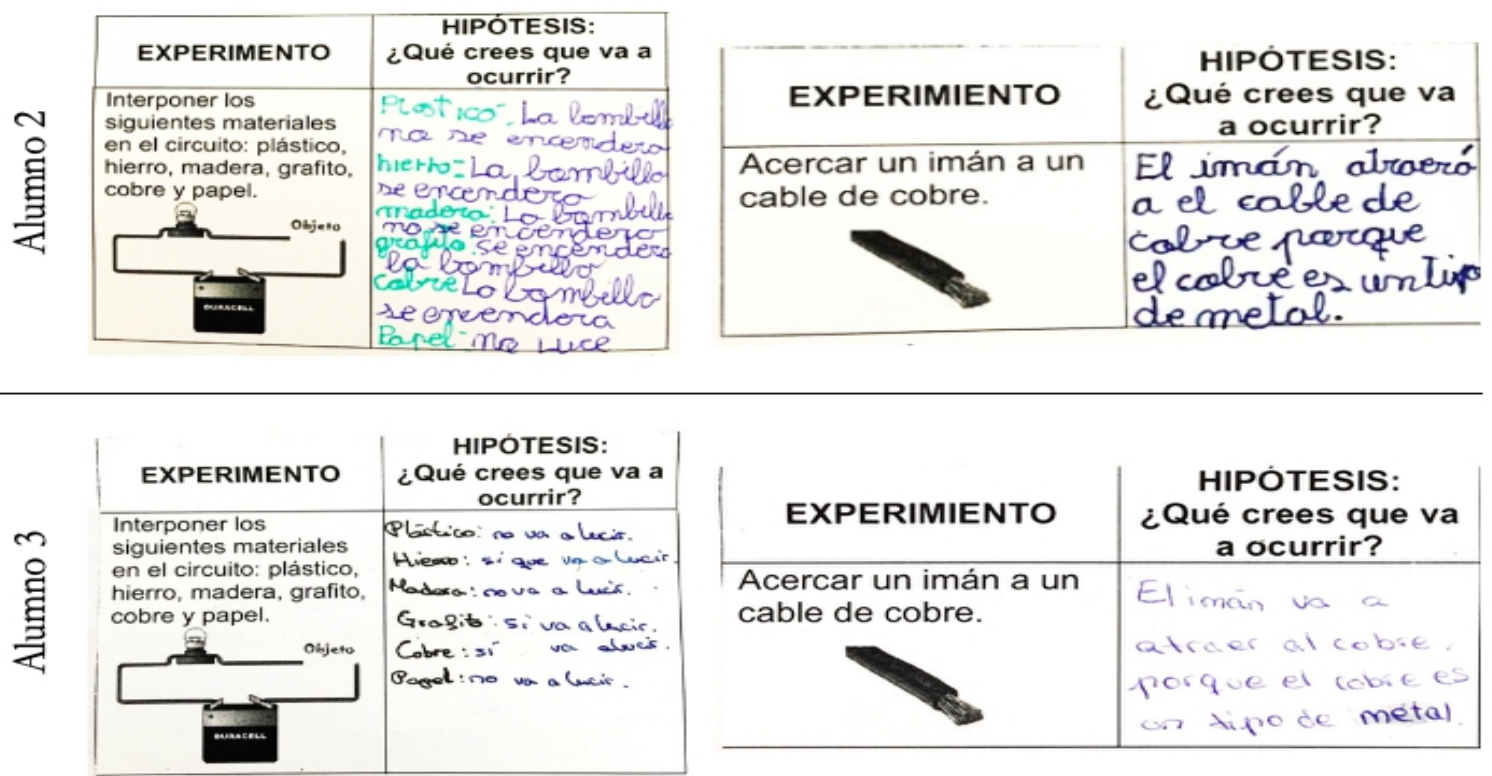

Figura 6. Ejemplificación del progreso de tres sujetos en la formulación de hipótesis en una actividad inicial y otra posterior.

Estos extractos del cuaderno de campo muestran cómo progresa sustancialmente la precisión en la formulación de hipótesis. Inicialmente, la mayoría del alumnado solo realizaba afirmaciones o mencionaba palabras sueltas, que indicaban qué es lo que creía que iba a suceder. Sin embargo, en la actividad posterior intentan justificar la hipótesis formulada, aunque esta justificación/explicación no sea necesariamente correcta desde el punto de vista científico. Este ejemplo refleja el comportamiento global de la muestra, tal como aparece en la tabla 6 tras realizar un recuento de los sujetos que en la actividad inicial realizaron al menos alguna afirmación a modo de hipótesis —es decir, aunque fueran simples, no eran palabras sueltas - y otro del alumnado que en la actividad posterior justificó, de alguna forma, la 
hipótesis formulada. Hay que destacar que el porcentaje de alumnado que proporcionó algún tipo de justificación en la actividad posterior es muy superior que en la actividad inicial.

Tabla 6. Recuento de sujetos con algún tipo de hipótesis en su actividad inicial —aunque muy reducidas— y en la actividad posterior — -hipótesis más justificadas-.

\begin{tabular}{|c|c|c|}
\hline Momento de la secuencia & $\begin{array}{c}\text { Frecuencia } \\
\text { absoluta }\end{array}$ & $\%$ \\
\hline Inicial & 41 & 33,9 \\
\hline Final & 110 & 90,9 \\
\hline
\end{tabular}

De acuerdo con estos resultados, la SEA iSTEAM resultó efectiva para mejorar la CMCT del alumnado desde las primeras actividades realizadas, al menos en relación con los contenidos abordados. Además, el porcentaje de consecución de la CMCT tanto por prototipo como en el total de la muestra indican valores altos de por sí, coincidiendo con resultados de otras investigaciones que muestran avances en el desarrollo competencial en áreas de ciencias y matemáticas mediante el uso del enfoque STEAM como, por ejemplo, Alsina y Salgado (2018) o Duban, Aydoğdu y Kolsuz (2018). Cabe agregar que el desarrollo competencial en las otras seis competencias claves fue alto y similar al alcanzado en la CMCT (Ortiz-Revilla 2020).

\section{Conclusiones}

El objetivo central de este trabajo era evaluar, usando la IBD, un modelo teórico para el diseño de SEA enmarcadas en la i-STEAM. Los resultados obtenidos para las dos primeras preguntas de investigación ofrecen evidencias a favor de la viabilidad del modelo teórico. A nivel de métodos, las modificaciones introducidas estuvieron enfocadas a una mayor concreción de las metodologías didácticas utilizadas —-más dirigidas en el caso de las indagaciones y menos en el caso del diseño de ingeniería-, pero no fueron sustituidas las propias metodologías dado que tanto docentes como alumnado trabajaron adecuadamente con ellas. Tampoco fueron sugeridas modificaciones en la concepción y/o construcción de los objetivos-representación ni del tipo y número de situaciones/actividades propuestas, aunque sí en su duración $\mathrm{y} / \mathrm{u}$ organización temporal. Por último, el nivel competencial alcanzado en todas las competencias es alto, sugiriendo que las elecciones teóricas y metodológicas han sido apropiadas para alcanzar el fin propuesto. Además, la ausencia de dificultades mayores por parte de los docentes que las implementaron en el contexto escolar regular - docentes que no tuvieron una capacitación específica- parecen también avalar el modelo.

Por otra parte, los resultados obtenidos aportan evidencias de la pertinencia de la iSTEAM para la mejora competencial integral del alumnado de Educación Primaria, si es implementado de forma coherente y fundamentada, coincidiendo con las escasas propuestas que, con una fundamentación más global, aparecen en la literatura (Chu et al. 2019).

El conocimiento producido por los estudios IBD es un conjunto de principios de diseño de aplicación general, desarrollado a partir de principios asumidos en la construcción de la innovación y basados en la literatura educativa, que son modificados, completados, o eliminados a partir de los datos empíricos sobre el grado en que las expectativas de aprendizaje se cumplieron. En el Anexo 1 se muestran algunos principios de diseño generales, emergentes del proceso seguido y evaluado en esta investigación, que pueden ser útiles para los docentes que quieran diseñar secuencias basadas en el modelo teórico propuesto.

Junto con las investigaciones de diseño aparece el concepto de generalización situada, basada en la similitud entre el contexto investigado y otros contextos, concepto análogo a la relación entre la muestra y la población en los estudios cuantitativos. mediante el reconocimiento de la 
existencia de problemas similares o compartidos, un profesor puede decidir utilizar los datos producidos por otros investigadores-docentes como evidencia para guiar sus acciones o juicios en su propia práctica. Por ello, esta investigación tomó un contexto real y se adaptó a sus características, en la expectativa que los resultados obtenidos puedan ser generalizables a otros contextos, que sean percibidos por los docentes como similares. En este sentido cabe señalar una limitación del trabajo: la evaluación competencial inicial del alumnado, evaluación que no fue realizada para reducir las interferencias con las clases. Sin embargo, en particular con la CMCT, consideramos que es una conjetura bien fundamentada suponer que este nivel inicial era bajo, tanto porque una enseñanza de las ciencias tradicional no la desarrolla como por los resultados de las evaluaciones de organismos oficiales (Ortiz-Revilla 2020).

Por último, insistimos en la relevancia de la IBD como herramienta metodológica para diseñar, implementar y evaluar propuestas didácticas que puedan ser efectivas para mejorar la educación y, en particular, la educación científica (Arriassecq et al. 2017, Guisasola et al. 2019), acordando con Burkhardt y Schoenfeld (2003) que para realizar una investigación educacional más útil, influyente y mejor financiada parece necesario moverse hacia este enfoque metodológico.

\section{Agradecimientos}

Agradecemos la colaboración del alumnado, maestras, maestros y equipo directivo del C.E.I.P. Fernando de Rojas (Burgos). Parte de esta investigación ha recibido financiación del Ministerio de Economía, Industria y Competitividad a través del proyecto EDU2017-89405-R.

\section{Referencias}

Aguilera Morales D., Martín-Páez T., Valdivia-Rodríguez V., Ruiz-Delgado A., Willians-Pinto L., Vílchez-González J. M., Perales-Palacios F. J. (2018) La enseñanza de las ciencias basada en indagación. Una revisión sistemática de la producción española. Revista de Educación 381, 259-284. https://doi.org/10.4438/1988-592X-RE-2017-381-388

Alsina Á., Salgado M. (2018) Land Art Math: una actividad STEAM para fomentar la competencia matemática en Educación Infantil. Edma 0-6: Educación Matemática en la Infancia 7(1), 1-11. http://www.edma0-6.es/index.php/edma0-6/article/view/48/37

Arriassecq I., Greca I. M., Cayul E. E. (2017) Secuencias de enseñanza y aprendizaje basadas en resultados de investigación: propuesta de un marco teórico para el abordaje de la teoría especial de la relatividad. Enseñanza de las Ciencias 35(1), 133-155. https://doi.org/10.5565/rev/ensciencias.1716

Artigue M. (1988) Ingénierie didactique. Recherches en Didactique des Mathématiques 9(3), 281-308. http://rdm.penseesauvage.com/Ingenierie-didactique.html

Astolfi J. P. (1999) El tratamiento didáctico de los obstáculos epistemológicos. Revista Educación y Pedagogía 11(25), 149-171. http://aprendeenlinea.udea.edu.co/revistas/index.php/revistaeyp/article/view/5863/ 5276

Bachelard G. (1938) La formation de l'esprit scientifique: contribution à une psychanalyse de la connaissance objective. París, Francia: Librairie Philosophique J. Vrin.

Barab S., Squire K. (2004) Design-based research: putting a stake in the ground. The Journal of the Learning Sciences 13(1), 1-14. https://doi.org/10.1207/s15327809jls1301_1

Burkhardt H., Schoenfeld A. H. (2003) Improving educational research: toward a more useful, more influential, and better-funded enterprise. Educational Researcher 32(9), 3-14. https://doi.org/10.3102/0013189X032009003 
Chu H-E., Martin S. N., Park, J. (2019) A theoretical framework for developing an intercultural STEAM program for Australian and Korean students to enhance science teaching and learning. International Journal of Science and Mathematics Education 17(7), 1251-1266. https://doi.org/10.1007/s10763-018-9922-y

Colucci-Gray L., Burnard P., Gray D., Cooke C. (2019) A critical review of STEAM (Science, Technology, Engineering, Arts, and Mathematics). En P. Thomson (Ed.), Oxford research encyclopedia of education (pp. 1-26). Oxford, Inglaterra: Oxford University Press.

Creswell J. W., Creswell J. D. (2018) Research design: qualitative, quantitative, and mixed methods approaches (5th ed.). Los Ángeles, CA: SAGE.

Duban N., Aydoğdu B., Kolsuz S. (2018) STEAM implementations for elementary school students in Turkey. Journal of STEM Arts, Crafts, and Constructions 3(2), 41-58. https://scholarworks.uni.edu/cgi/viewcontent.cgi?article=1030\&context=journalstem-arts

Duit R. (2006) La investigación sobre enseñanza de las ciencias. Un requisito imprescindible para mejorar la práctica educativa. Revista Mexicana de Investigación Educativa 11(30), 741 770.

http://comie.org.mx/revista/v2018/rmie/index.php/nrmie/article/view/715/715

Fournier M. V., Garnier L. A. (1990) Fórmula abreviada para la transformación de puntajes de variables a una misma escala. Revista Latinoamericana de Psicología 22(3), 421-426.

Godino J. D., Batanero C., Contreras Á, Estepa A., Lacasta E., Wilhelmi M. R. (2013) Didactic engineering as design-based research in mathematics education. Trabajo presentado en el Eighth Congress of European Research in Mathematics Education (CERME 8), Manavgat, Turquía.

Greca I. M., Ataíde A. R. P. (2019) Theorems-in-action for problem-solving and epistemic views on the relationship between physics and mathematics among preservice physics teachers. En G. Pospiech, M. Michelini y B-S. Eylon (Eds.), Mathematics in physics education (pp. 153-173). Cham, Suiza: Springer.

Guisasola J., Zuza K., Ametller J., Gutiérrez-Berraondo J. (2017) Evaluating and redesigning teaching learning sequences at the introductory physics level. Physical Review Physics Education Research 13(2) 1-14. https://doi.org/10.1103/PhysRevPhysEducRes.13.020139

Guisasola J., Zuza K., Sagastibeltza M. (2019) Una propuesta de diseño y evaluación de secuencias de enseñanza-aprendizaje en Física: el caso de las leyes de Newton. Revista de Enseñanza de la Física 31(2) https://revistas.unc.edu.ar/index.php/revistaEF/article/view/26948/28600

Juuti K., Lavonen J. (2006) Design-based research in science education: one step towards methodology. Nordic Studies in Science Education 2(2) 54-68. https://doi.org/10.5617/nordina.424

Kang N-H. (2019) A review of the effect of integrated STEM or STEAM (science, technology, engineering, arts, and mathematics) education in South Korea. Asia-Pacific Science Education 5(6), 1-22. https://doi.org/10.1186/s41029-019-0034-y

Laudan L. (1977) Progress and its problems. Berkeley, CA: University of California Press.

Laudan L. (1984) Science and values: the aims of science and their role in scientific debate. Berkeley, CA: University of California Press. 
Martinand J. L. (1986) Connaître et transformer la matière. Berna, Suiza: Peter Lang.

Martin-Hansen L. (2002) Defining inquiry: exploring the many types of inquiry in the science classroom. The Science Teacher 69(2), 34-37. https://www.jstor.org/stable/24154746

Massot Lafon I., Dorio Alcaraz I., Sabariego Puig M. (2004) Estrategias de recogida y análisis de la información. En R. Bisquerra Alzina (Coord.), Metodología de la investigación educativa (pp. 329-366). Madrid, España: La Muralla.

Méheut M., Psillos D. (2004) Teaching-learning sequences: aims and tools for science education research. International Journal of Science Education 26(5), 515-535. https://doi.org/10.1080/09500690310001614762

Ortiz-Revilla J. (2018) ¿Cómo diseñar un prototipo de iluminación para mi sala de estudio? En I. M. Greca y J. Á. Meneses Villagrá (Eds.), Proyectos STE AM para la Educación Primaria. Fundamentos y aplicaciones prácticas (pp. 163-193). Madrid, España: Dextra.

Ortiz-Revilla J. (2020) El desarrollo competencial en la Educación Primaria: efectos de una propuesta STEAM integrada (Tesis doctoral). Recuperada de https://riubu.ubu.es/handle/10259/5521

Ortiz-Revilla J., Greca I. M. (2019) La evaluación del desarrollo competencial escolar: propuesta de un instrumento. En M. C. Pérez-Fuentes (Ed.), Innovación docente e investigación en educación (pp. 365-374). Madrid, España: Dykinson.

Ortiz-Revilla J., Greca I. M., Adúriz-Bravo A. (2021) Conceptualización de las competencias: revisión sistemática de su investigación en Educación Primaria. Profesorado. Revista de Currículum y Formación del Profesorado (en prensa).

Ortiz-Revilla J., Greca I. M., Adúriz-Bravo A. (2018) La Educación STEAM y el desarrollo competencial en la Educación Primaria. En I. M. Greca y J. Á. Meneses Villagrá (Eds.), Proyectos STE AM para la Educación Primaria. Fundamentos y aplicaciones prácticas (pp. 41-54). Madrid, España: Dextra.

Ortiz-Revilla J., Greca I. M., Arriassecq I. (2018) Construcción de un marco teórico para el enfoque STEAM en la Educación Primaria. En C. Martínez Losada y S. García Barros (Eds.), 28 Encuentros de Didáctica de las Ciencias Experimentales. Iluminando el cambio educativo (pp. 823-828). A Coruña, España: Universidade da Coruña.

Plomp T. (2013) Educational design research: an introduction. En T. Plomp y N. Nieveen (Eds.), Educational design research. Part A: an introduction (pp. 10-51). Enschede, Países Bajos: SLO.

del Rincón Igea D., Arnal Agustín J., Latorre Beltrán A., Sans Martín A. (1995) Técnicas de investigación en ciencias sociales. Madrid, España: Dykinson.

Spradley J. P. (2016) Participant observation (2nd ed.). Long Grove, IL: Waveland Press.

Vergnaud G. (1990) La théorie des champs conceptuels. Recherches en Didactique des Mathématiques 10(2.3), 133-170. http://rdm.penseesauvage.com/La-theorie-deschamps-conceptuels.html

Yakman G. (2008) ST $\sum @$ M education: an overview of creating a model of integrative education. PATT-17 and PATT-19 Proceedings, 335-358. Recuperado de https:/ /www.iteea.org/File.aspx?id $=86752 \& v=75 \mathrm{ab076a}$

Zeidler D. L. (2016) STEM education: a deficit framework for the twenty first century? A sociocultural socioscientific response. Cultural Studies of Science Education 11(1), 11-26. https://doi.org/10.1007/s11422-014-9578-z 
Anexo 1. Principios de diseño de SEA iSTEAM basadas en el modelo teórico de Ortiz-Revilla, Greca y Arriassecq (2018)

Escoger una temática curricular —Ciencias, Matemáticas, Artes, etc.—

$\downarrow$

Proponer una situación problemática relevante para el alumnado que permita abordar aspectos del tema empleando la metodología del diseño de ingeniería $\downarrow$

Escoger los contenidos de las diversas áreas curriculares necesarios para resolver la situación problemática y seleccionar los Estándares de Aprendizaje Evaluables (EAE)

$$
\downarrow
$$

Identificar —a través de literatura, test, etc.—y seleccionar representaciones del alumnado en relación con los contenidos escogidos y construir los objetivos-representación

\section{$\downarrow$}

Identificar, junto al alumnado, problemas investigables de la situación problemática inicial que puedan abordarse de forma independiente mediante indagaciones y que se correspondan con los contenidos escogidos de las diferentes áreas. Estos problemas deben ofrecer variedad de situaciones para enriquecer los esquemas mentales del alumnado en cuanto a los contenidos abordados

$$
\downarrow
$$

Evaluar el desarrollo competencial del alumnado teniendo en cuenta la consecución de los EAE

$$
\downarrow
$$

Si la evaluación no ha resultado satisfactoria volver a - elegir una de las alternativas, a partir de las evidencias recogidas-
Revisar si la situación es interesante; si desde el punto de vista conceptual se encuentra en la zona de desarrollo proximal del alumnado; si existe adecuación entre la complejidad y los recursos - tiempo, materiales, etc.disponibles

Revisar si las representaciones seleccionadas se corresponden con el perfil específico del alumnado

Revisar si los problemas investigables son apropiados; si los contenidos están bien secuenciados; si se necesita más tiempo; si se necesita más explicación o dirección

Revisar si la evaluación es coherente con la metodología y con los EAE 